\title{
A Case of Crohn's Disease Admitted with Acute Abdomen
}

\author{
Yeliz Cagan Appak ${ }^{1 *}$, Guzide Dogan ${ }^{1}$, Serdar Tarhan $^{2}$, Semin Ayhan $^{3}$, Erhun Kasırga ${ }^{1}$
}

\begin{abstract}
Crohn's disease is an inflammatory disorder and may affect any part of gastrointestinal tract. The signs and symptoms of Crohn's disease are similar to many other abdominal disorders. Crohn's disease does not typically present as an acute abdomen. However, Crohn's disease should be considered in the differential diagnosis of patients with abdominal pain, especially relevant to patients with a long history of vague abdominal complaints and those with unexplained weight loss or growth retardation. In this case report, we present such a patient of acute abdomen admitted to us after appendectomy and diagnosed Crohn's disease finally.
\end{abstract}

Key words: Crohn's disease; abdomen; child

\section{Introduction}

Crohn's disease (CD) is a disorder that can involve the whole gastrointestinal system (GIS) and that can show diversified clinical symptoms (1). Therefore, it may be difficult to make differential diagnosis in case of CD. Concerning the classical CD, typical story, right lower quadrant tenderness or mass, characteristic radiographic symptoms may ease diagnosis (2). For CD patients who do not come with classical symptoms, acute abdominal symptoms may lead to confusion in diagnosis and to unnecessary surgical interventions. In this report, we present the case of a 17-year old male patient who came with acute abdominal clinic and appendectomy was applied, but when the symptoms did not regress, he was considered and diagnosed to have CD.

\section{Case Presentation}

The seventeen year-old male patient reportedly applied to emergency service for having abdominal pain for the last two weeks that was more explicit in the abdominal right lower quadrant and epigastric region. The patient was evaluated by the department of general surgery and mesentery lymphadenitis was detected; as the abdominal pain lessened in the followup, the patient was discharged with ciprofloxacin and ornidazole treatment. One week later he applied to the emergency service again with the increase in abdominal pain. In the physical examination; weight: $46.8 \mathrm{~kg}(<3 \mathrm{p})$, length: $164 \mathrm{~cm}(3-10 \mathrm{p})$; the patient had epigastric region and abdominal right lower quadrant tenderness and rebound, and was hospitalized by the department of general surgery.
In the abdominal ultrasonography (USG) free fluid around of cecum was detected and the patient was followed on suspicion of perforated appendicitis. Computerized tomography (CT) of the abdomen revealed wall thickening in the part descending to duodenum and indicated duodenitis. The patient was operated by the department of general surgery, duodenum perforation was not detected and appendectomy was applied. The abdominal pain repeated 10 days after application of appendectomy and following his application to general surgery, he was directed to pediatric gastroenterology. When the patient applied to us, his physical examination showed that he had prevalent abdominal pain more explicit in both of the lower quadrants and he lost 5 kilos in one month. In the repeated $\mathrm{CT}$, mucosal thickening was seen in the terminal ileum, intermittent wall thickening was seen in the jejunum and ileum, and free fluid in pelvis (Figure 1). White blood cell count of the patient: $13700 / \mathrm{Ul}$, hemoglobin: $13,7 \mathrm{~g} / \mathrm{dl}$, platelet count: $576000 \quad / \mathrm{Ul}$, aspartate aminotransferase: $17 \mathrm{U} / \mathrm{L}$, alanine aminotransferase: $25 \mathrm{U} / \mathrm{L}$, total protein: $7 \mathrm{~g} / \mathrm{dL}$, albumin: $4 \mathrm{~g} / \mathrm{dl}, \mathrm{C}-$ reactive protein: $2,3 \mathrm{mg} / \mathrm{dl}$ (normal: $0-0,5 \mathrm{mg} / \mathrm{dl}$ ), erythrocyte sedimentation rate: $46 \mathrm{~mm} / \mathrm{s}$, vitamin B12: $230 \mathrm{pg} / \mathrm{mL}$, ferritin: $176 \mathrm{ng} / \mathrm{mL}$, folic acid: $18 \mathrm{ng} / \mathrm{mL}$, ANCA: negative. In the upper GIS endoscopy, apparent aphthous and convoluted ulcer, fragile and granular mucosa was observed in the duodenum second part and there were also regions where usual mucosa could be observed (Figure 2).

Received: 27-11-2015, Accepted 29-12-2015, Available Online 15-02-2016

1 Celal Bayar University School of Medicine, Department of Pediatric Gastroenterology, Manisa-Turkey

2 Celal Bayar University School of Medicine, Department of Radiology, Manisa-Turkey

3 Celal Bayar University School of Medicine Department of Pathology, Manisa-Turkey

*Corresponding Author: Yeliz Cagan Appak E-mail: yelizcagan@yahoo.com 
The colonoscopy of the patient revealed partly faintness in mucosa, nodularity and granular pattern in the colon and there were skin tag and haemorrhoidal formations in the anal region.

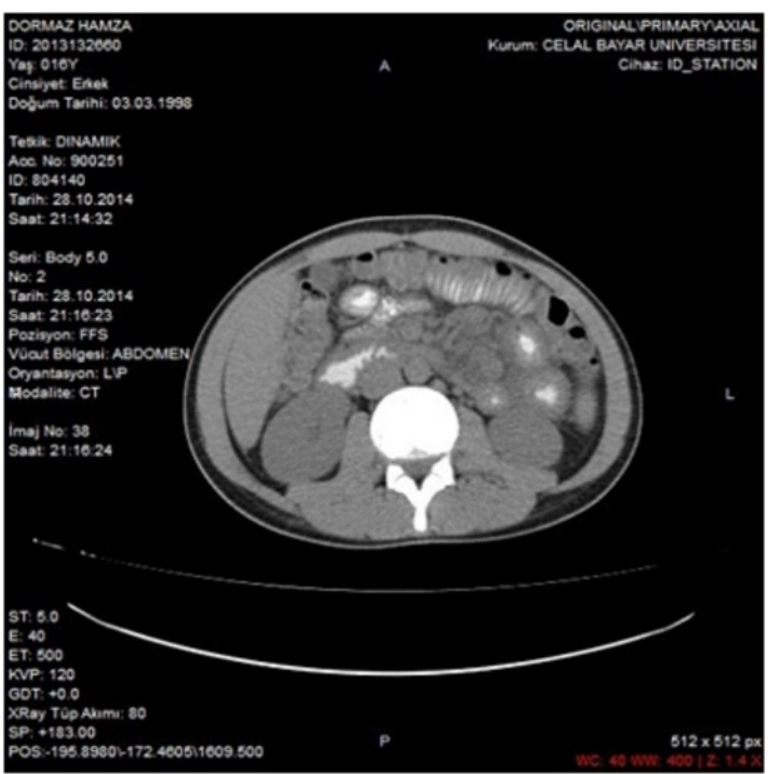

Figure 1. Increase in wall thickness of the patient in jejunum and ileum presented by abdomen CT.

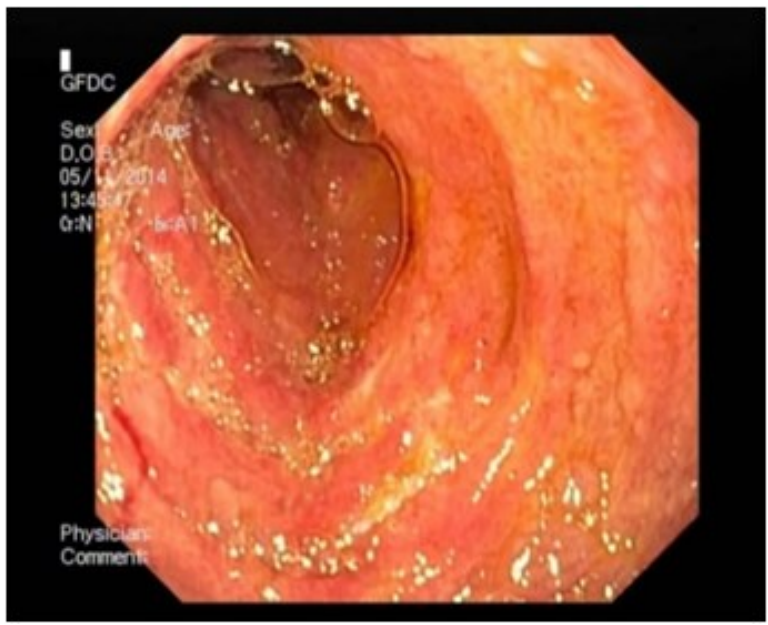

Figure 2. Apparent aphthous and convoluted ulcers, fragile and granular mucosa in the second part of duodenum as presented by the upper GIS endoscopy.

The patient was reported to have edematous colon mucosa with the result of colonoscopic histopathology and as a result of the upper GIS endoscopy pathology edema was seen in the lamina propria of duodenum, little number of leukocyte with polymorphic nuclei infiltration and the findings were evaluated nonspecifically. The patient's abdominal pain continued and he was reported to have normal appendix vermiformis as the pathology result of appendectomy material, and together with the clinic and laboratory symptoms he was considered to have CD. The patient started to receive methylprednisolone and mesalamin treatment and he was supported by enteral feeding. The clinical symptoms of the patient regressed and azathioprine treatment was added and the steroid treatment was gradually cut down and terminated. The patient put on weight in the follow-up and his complaints did not repeat.

\section{Discussion}

$\mathrm{CD}$ is a chronic, recurrent, inflammatory disorder characterized by transmural inflammation that may affect any part of the GIS, mostly involving ileum and colon (1). The clinical symptoms of the CD varies greatly according to its location and severity of the disease and may be confused with the symptoms of many abdomen based diseases such as tuberculosis, ulcerative colitis and irritable bowel disorder. Although there are many examinations used for diagnosis of $\mathrm{CD}$, there is no gold standard method for establishing a differential diagnosis (2). Therefore, diagnosis of $\mathrm{CD}$ to differ it from other diseases is difficult. While non-caseating granuloma indicates $\mathrm{CD}$, its non-existence does not exclude the diagnosis. There is no histopathological symptom specific to CD and the symptoms detected can also be seen in other disorders. The $\mathrm{CD}$ diagnosis is given when the story and the physical examination symptoms are supported by detectable laboratory, radiological, serologic or pathological data (3). The duodenal lesions in the upper GIS endoscopy, colonoscopy findings, wall thickening at jejunum, ileum and terminal ileum revealed by abdominal $\mathrm{CT}$, loss of weight, height percentile retardation and the highness of acute phase reactants in our patient led to consideration of diagnosis of $\mathrm{CD}$ and the patient gave response to the medical treatment.

The antibiotics treatment that the surgery department gave at the initial application of our patient might have provided a temporary relief. In perforated appendicitis, especially as the disorder prolongs, the standard method of appendectomy is inadequate in preventing the development of complications such as intestinal fistule, peritonitis, intraabdominal abscess and acute intestinal obstruction (4). In the recurrent application of the patient, he received diagnosis of urgent surgical indication probably with the consideration that the patient had right lower quadrant tenderness, rebound and the USG was interpreted as perforated appendicitis.

Concerning the distinctive diagnosis of abdominal pain, CD should be considered especially for those patients who have long term indeterminate abdominal complaints, loss of weight and growth retardation. Abdominal pain may accompany $\mathrm{CD}$, but typically acute abdominal symptoms are not frequent. For this reason, patients who come with acute abdominal 
symptoms and other symptoms that may recall $\mathrm{CD}$ are soft, may receive late diagnosis in the aftermath of many examinations and unnecessary surgical interventions $(3,5)$. As is the case for our patient, loss of weight, height percentile retardation, together with the story and physical examination are cautionary with regards to chronic diseases and especially for children, these features should lead to consideration of further examination before surgery.

There is the case of a fourteen-year old male patient in the literature who, following recurrent application to emergency service due to abdominal pain and vomiting, was considered to have perforated appendicitis. After he received resection for reasons of dilatation, increase in thickness and mass image in distal ileum in laparotomy, he received the diagnosis of CD (5). For patients who apply with CD complications such as stricture, obstruction and perforation in GIS in the first diagnosis, acute abdomen symptoms can be observed and the diagnosis can be made with difficulty. The literature includes cases of patients who apply with acute abdomen symptoms and considered as intestinal obstruction after application, and following surgery, terminal ileum involvement is in the foreground and the patients receive $\mathrm{CD}$ diagnosis histopathologically (6). It was seen that acute abdomen symptoms in our patient were not related with $\mathrm{CD}$ complications, and perforation, fistule, stricture was not detected, and the symptoms lessened with medical treatment. The abdomen pain of the patient was connected to small bowel involvement.

Inflammatory disorders of the right lower quadrant can mimic CD. There is also a case who had recurrent abdominal pain and was taken under treatment for having $C D$ and whose abdominal pain repeated while under treatment and consequently received diagnosis of chronic non-granulomatous appendicitis in the literature (7). Therefore, as in this case, appendicitis can receive a wrong $\mathrm{CD}$ diagnosis and $\mathrm{CD}$ may cause confusion in diagnosis including appendix or can be seen only with appendix involvement (8).

In $0.5-4 \%$ of $C D$ patients, duodenum involvement is observed $(9,10)$. In our patient the lesions could be seen in in the second part of duodenum with the upper GIS endoscopy and this helped in diagnosing CD. The patients having duodenal involvement very rarely have duodenal stricture requiring surgical treatment (10).
Therefore, the follow-up of our patient is important with regards to long term complications.

Consequently, because CD can involve many parts of the GIS and because its symptoms can be confused with many other disorders the diagnosis of $\mathrm{CD}$ becomes difficult. Especially for patients, like our patient, who have acute abdomen like recurrent abdominal pain, CD should definitely be taken into account in the definitive diagnosis.

Conflict of Interest: The authors declare no potential conflicts of interest with respect to the research, authorship, and/or publication of this article.

\section{References}

1. Podolsky DK. Inflammatory bowel disease. N Engl J Med 2002; 347: 417-429.

2. Stange EF, Travis SP, Vermeire S, Beglinger C, Kupcinkas L, Geboes K, et al. European evidence based consensus on the diagnosis and management of Crohn's disease: definitions and diagnosis. Gut 2006; 55; 1-15.

3. Laass MW, Roggenbuck D, Conrad K. Diagnosis and classification of Crohn's disease. Autoimmun Rev 2014; 13: $467-471$

4. Shiryajev YN, Volkov NN, Kashintsev AA, Chalenko MV, Radionov YV. Appendectomy and resection of the terminal ileum with secondary severe necrotic changes in acute perforated appendicitis. Am J Case Rep 2015; 16: 37-40.

5. Judge J, Giordano BP, English J. Crohn's disease masquerading as an acute abdomen. J Pediatr Health Care 2014; $28: 444-450$.

6. Gupta M, Goyal S, Goyal R. Crohn's disease presenting as acute abdomen: Report of two cases. N Am J Med Sci 2011; 3: 209-211

7. Shaoul R, Rimar Y, Toubi A, Mogilner J, Polak R, Jaffe M. Crohn's disease and recurrent appendicitis: a case report. World J Gastroenterol 2005 ; 11: 6891-6893.

8. Hsu WF, Wu CS, Wu JM, Chung CS. Ileal Crohn's disease with perforation misdiagnosed as ruptured appendicitis: a case report. J Formos Med Assoc 2013; 112: 652-653.

9. Nugent FW, Roy MA. Duodenal Crohn's disease: an analysis of 89 cases. Am J Gastroenterol 1989; 84: 249254

10. Racz JM, Davies W. Severe stricturing Crohn's disease of the duodenum: A case report and review of surgical options. Int J Surg Case Rep 2012; 3: 242-245. 